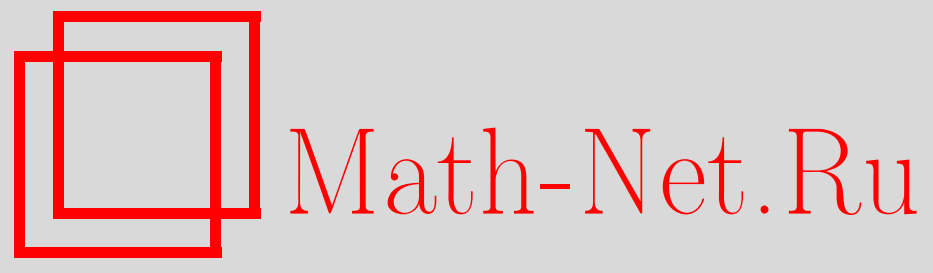

Р. Х. Макаова, Краевая задача для гиперболического уравнения третьего порядка с вырождением порядка внутри области, Вестн. Сам. гос. техн. ун-та. Сер. Физ.-мат. науки, 2017, номер 4, 651664

DOI: https://doi.org/10.14498/vsgtu1574

Использование Общероссийского математического портала MathNet.Ru подразумевает, что вы прочитали и согласны с пользовательским соглашением

http://www . mathnet.ru/rus/agreement

Параметры загрузки:

IP : 18.208 .226 .222

26 апреля 2023 г., $14: 23: 59$

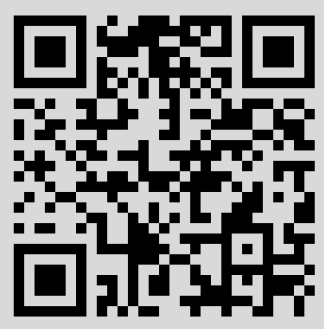


Вестн. Сам. гос. техн. ун-та. Сер. Физ.-мат. науки. 2017. Т. 21, № 4 . С. $651-664$ ISSN: 2310-7081 (online), 1991-8615 (print)

УДК 517.956.326

\title{
Краевая задача для гиперболического уравнения третьего порядка с вырождением порядка внутри области
}

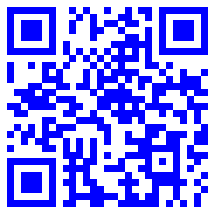

\author{
P. Х. Макаова \\ Институт прикладной математики и автоматизации - \\ филиал Федерального государственного бюджетного научного учреждения \\ «Федеральный научный центр «Кабардино-Балкарский научный центр \\ Российской академии наук», \\ Россия, 360000, Нальчик, ул. Шортанова, 89 а.
}

\section{Аннотация}

Исследуется краевая задача для гиперболического уравнения третьего порядка с вырождением типа внутри смешанной области. Рассматриваемое уравнение в положительной части области совпадет с уравнением Аллера, которое является уравнением псевдопараболического типа. А в отрицательной части области - с вырождающимся гиперболическим уравнением первого рода, частным случаем которого является уравнение Бицадзе-Лыкова. Доказана теорема существования и единственности решения. Единственность решения задачи доказана с помощью метода Трикоми. Из функциональных соотношений, принесенных на линию вырождения порядка из положительной и отрицательной частей области, приходим к уравнению Вольтерра второго рода типа свертки относительно следа производной искомого решения. Путем применения метода интегрального преобразования Лапласа решение интегрального уравнения находится в явном виде. Далее решение исследуемой задачи выписывается в явном виде как решение второй краевой задачи для уравнения Аллера в положительной части области и как решение задачи Коши для вырождающегося гиперболического уравнения первого рода в отрицательной части области.

Ключевые слова: краевая задача, гиперболическое уравнение третьего порядка, уравнение Аллера.

Получение: 27 октября 2017 г. / Исправление: 11 декабря 2017 г. / Принятие: 18 декабря 2017 г. / Публикация онлайн: 28 декабря 2017 г.

\section{Научная статья}

ə @(;) Контент публикуется на условиях лицензии Creative Commons Attribution 4.0 International (https://creativecommons.org/licenses/by/4.0/deed.ru)

\section{Образец для цитирования}

Мак а о в а Р. Х. Краевая задача для гиперболического уравнения третьего порядка с вырождением порядка внутри области // Вестн. Сам. гос. техн. ун-та. Сер. Физ.-мат. науки, 2017. Т. 21, № 4. C. 651-664. doi: 10.14498/vsgtu1574.

\section{Сведения об авторе}

Рузанна Хасанбиевна Макаова (1) http://orcid.org/0000-0003-4095-2332

младший научный сотрудник; отд. уравнений смешанного типа;

e-mail: makaova.ruzanna@mail.ru 
Введение. В евклидовой плоскости точек $(x, y)$ рассматривается уравнение вида

$$
0= \begin{cases}u_{y}-a u_{x x}-b u_{x x y}, & y>0 \\ (-y)^{m} u_{x x}-u_{y y}-c(-y)^{(m-2) / 2} u_{x}, & y<0\end{cases}
$$

где $a, b, m$ - заданные положительные числа; $|c| \leqslant m / 2 ; u=u(x, y)$ - искомая действительная функция независимых переменных $x$ и $y$.

Уравнение (1) при $y>0$ совпадает с уравнением Аллера [1]:

$$
\frac{\partial u}{\partial y}=\frac{\partial}{\partial x}\left(a \frac{\partial u}{\partial x}+b \frac{\partial^{2} u}{\partial x \partial y}\right)
$$

а при $y<0-$ с вырождающимся гиперболическим уравнением первого рода [2]:

$$
(-y)^{m} u_{x x}-u_{y y}-c(-y)^{(m-2) / 2} u_{x}=0 .
$$

Уравнение (2) относят к уравнениям псевдопарабролического типа [3], хотя оно является уравнением гиперболического типа. При определенных допущениях уравнение (2) описывает движение влаги в капиллярно-пористых средах [4] и его решение «интерпретируется как влажность почвы с коэффициентом диффузии $a$ и коэффициентом влагопроводности $b$ в точке $x$ почвенного слоя $0 \leqslant x \leqslant r$ в момент времени $y \in[0, T] \gg[5]$. Изучению краевых задач для уравнений третьего порядка псевдопараболического типа, в частности для уравнения Аллера, посвящены такие работы, как [6-12].

Уравнение (3) является уравнением гиперболического типа и при $m=2$ его называют уравнением Бицадзе-Лыкова [13-15]. При $c=0$ уравнение (3) переходит в уравнение Геллерстедта, которое находит применение к отыскании оптимальной формы плотины прорези [16]. В работах [17, 18] были изучены первая и вторая задачи Дарбу для уравнения (3). Исследованию различных краевых задач для вырождающихся гиперболических уравнений посвящены работы $[2,19-22]$.

Постановка задачи и полученный результат. Пусть $\Omega^{+}=\{(x, y)$ : $0<x<r, 0<y<T\}$. Через $\Omega^{-}$обозначим область, ограниченную характеристиками урав-

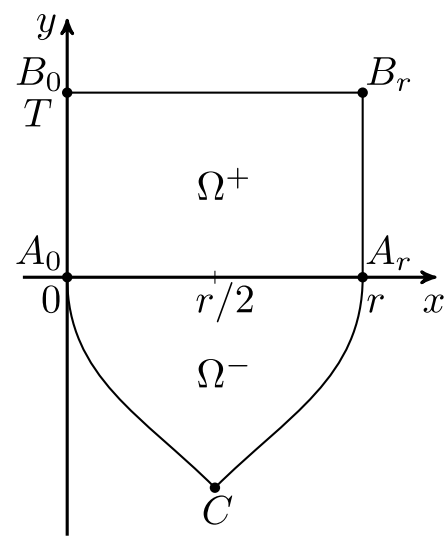
нения (3):

$$
\begin{aligned}
& A_{0} C: x-\frac{2}{m+2}(-y)^{(m+2) / 2}=0, \\
& A_{r} C: x+\frac{2}{m+2}(-y)^{(m+2) / 2}=r,
\end{aligned}
$$

выходящими из точек $A_{0}=(0,0), A_{r}=(r, 0)$ и пересекающимися в точке

$$
C=\left(r / 2,-[(m+2) r / 4]^{2 /(m+2)}\right),
$$

и отрезком $A_{0} A_{r}=\{(x, 0): 0<x<r\}$. Пусть $B_{0}=(0, T), B_{r}=(r, T)$ и $A_{0} B_{0}=\{(0, y):$ $0<y<T\}, A_{r} B_{r}=\{(r, y): 0<y<T\} ;$ $\Omega=\Omega^{+} \cup \Omega^{-} \cup\left(A_{0} A_{r}\right)$. 
ОПРедЕлениЕ. Регулярным в области $\Omega$ решением уравнения $(1)$ назовем функцию $u=u(x, y)$ такую, что $u \in C(\bar{\Omega}) \cap C^{1}(\Omega) \cap C^{2}\left(\Omega^{-}\right), u_{x x}, u_{x x y} \in C\left(\Omega^{+}\right)$, $u_{x x}(x, 0), u_{x x y}(x, 0) \in L_{1}\left(A_{0} A_{r}\right)$, и удовлетворяющую уравнению $(1)$.

Исследуется следующая

ЗАДАчА. Найти регулярное в области $\Omega$ решение уравнения (1) из класса $u_{x} \in C\left(\overline{A_{0} B_{0}} \cup \overline{A_{r} B_{r}}\right)$, удовлетворяющее следующим условиям:

$$
\begin{aligned}
& u_{x}(0, y)=\nu(y), \quad 0 \leqslant y \leqslant T, \\
& u_{x}(r, y)=\nu_{r}(y), \quad 0 \leqslant y \leqslant T, \\
& u_{C A_{r}}=h_{r}(x), \quad r / 2 \leqslant x \leqslant r,
\end{aligned}
$$

где $\nu(y), \nu_{r}(y) \in C^{1}[0, T], h_{r}(x) \in C^{3}[0, r]$.

\section{Имеет место следующая}

Теорема. Регулярное решение задачи (4)-(6) для уравнения (1) существует и оно единственно.

Доказ а тельство. Единственность решения. Положим

$$
\begin{gathered}
u(x, 0)=\varphi(x), \quad 0 \leqslant x \leqslant r, \\
u_{y}(x, 0)=\psi(x), \quad 0<x \leqslant r .
\end{gathered}
$$

Из $(2)$, переходя к пределу при $y \rightarrow+0$ с учетом $(7)$ и $(8)$, находим функциональное соотношение между функциями $\varphi(x)$ и $\psi(x)$, принесенное из области $\Omega^{+}$на линию $y=0$, в виде

$$
\psi(x)-a \varphi^{\prime \prime}(x)-b \psi^{\prime \prime}(x)=0
$$

Проинтегрировав равенство (9) дважды в пределах от $x$ до $r$, получим

$$
a \varphi(x)+b \psi(x)-\int_{r}^{x}(x-\xi) \psi(\xi) d \xi=a \varphi(r)+b \psi(r)-(r-x)\left[a \varphi^{\prime}(r)+b \psi^{\prime}(r)\right]
$$

Пользуясь формулой нахождения производной по заданному направлению [23, п. 184], запишем следующую формулу для функции $h_{r}(x)$ :

$$
h_{r}^{\prime}(x)=u_{y}(x, y) y_{x}+u_{x}(x, y), \quad \forall(x, y) \in C A_{r}
$$

Для любых точек $(x, y) \in C A_{r}$ из $(11)$ получаем

$$
u_{y}(x, y)=\left[h_{r}^{\prime}(x)-u_{x}(x, y)\right]\left[\frac{m+2}{2}(r-x)\right]^{m /(m+2)} .
$$

Так как $u_{y}(x, 0) \in L_{1}\left(A_{0} A_{r}\right)$, из формулы $(12)$ верно, что $u_{y}(r, 0)=\psi(r)=0$. Тогда с учетом условий согласования

$$
\varphi(r)=h_{r}(r), \quad \varphi^{\prime}(r)=\nu_{r}(0), \quad \psi^{\prime}(r)=\nu_{r}^{\prime}(0)
$$

равенство (10) можно переписать в виде

$$
\varphi(x)=\frac{1}{a} D_{r x}^{-2} \psi(\xi)-\frac{b}{a} \psi(x)+h_{r}(r)-(r-x)\left[\nu_{r}(0)+\frac{b}{a} \nu_{r}^{\prime}(0)\right] .
$$


Через $D_{r x}^{\gamma}$ обозначим оператор дробного интегро-дифференцирования в смысле Римана-Лиувилля по переменной $x$ порядка $\gamma$ с началом в точке $r$ и с концом в точке $x$, определяемый следующим образом [16]:

$$
\begin{gathered}
D_{r x}^{\gamma} v(\xi)=\frac{\operatorname{sign}(x-r)}{\Gamma(-\gamma)} \int_{r}^{x} \frac{v(\xi) d \xi}{|x-\xi|^{\gamma+1}}, \quad \gamma<0 ; \\
D_{r x}^{\gamma} v(\xi)=v(x), \quad \gamma=0 ; \\
\left.\left.D_{r x}^{\gamma} v(\xi)=\operatorname{sign}(x-r) \frac{d^{n}}{d x^{n}} D_{r x}^{\gamma-n} v(\xi), \quad \gamma \in\right] n-1, n\right], n=1,2, \ldots,
\end{gathered}
$$

где $Г(z)$ - гамма-функция Эйлера.

Рассмотрим интеграл

$$
I=\int_{0}^{r} u(x, 0) u_{y}(x, 0) d x=\int_{0}^{r} \varphi(x) \psi(x) d x .
$$

Из (13) и (14) при однородных краевых условиях (4)-(6) получим

$$
I=\frac{1}{a} \int_{0}^{r} \psi(x) D_{r x}^{-2} \psi(\xi) d x-\frac{b}{a}\|\psi(x)\|_{0}^{2},
$$

где $\|f\|_{0}^{2}=\int_{0}^{r} f^{2}(x) d x-$ норма в $L_{2}[0, r]$.

Интеграл, стоящий в правой части равенства (15), перепишем в виде

$$
\begin{aligned}
\left(\psi, D_{r x}^{-2} \psi\right)_{0}=\int_{0}^{r} \psi(x) \int_{x}^{r}(\xi-x) \psi(\xi) d \xi d x & = \\
& =\int_{0}^{r} \psi(\xi) d \xi \int_{0}^{\xi}(\xi-x) \psi(x) d x=\left(\psi, D_{0 x}^{-2} \psi\right)_{0}
\end{aligned}
$$

откуда находим

$$
\left(\psi, D_{0 x}^{-2} \psi\right)_{0}=\int_{0}^{r}(r-x) \psi(x) d x \int_{0}^{r} \psi(x) d x-\int_{0}^{r}\left(\int_{0}^{x} \psi(\xi) d \xi\right)^{2} d x
$$

где $(f, g)_{0}=\int_{0}^{r} f(x) g(x) d x$ - скалярное произведение в пространстве $L_{2}[0, r]$.

Далее проинтегрируем уравнение (2) по переменной $x$ в пределах от 0 до $r$. С учетом (4) и (5) имеем

$$
\int_{0}^{r} u_{y}(x, y) d x=\int_{0}^{r} \frac{\partial}{\partial x}\left[a \frac{\partial u}{\partial x}+b \frac{\partial^{2} u}{\partial x \partial y}\right] d x=a \nu_{r}(y)+b \nu_{r}^{\prime}(y)-a \nu(y)-b \nu^{\prime}(y)
$$

Отсюда, когда условия (4) и (5) однородные, находим

$$
\int_{0}^{r} u_{y}(x, y) d x=0, \quad 0 \leqslant y \leqslant T .
$$


Следовательно, из (16) с учетом условия (17) получаем оценку

$$
\left(\psi, D_{r x}^{-2} \psi\right)_{0}=\left(\psi, D_{0 x}^{-2} \psi\right)_{0}=-\left\|\int_{0}^{x} \psi(\xi) d \xi\right\|_{0}^{2} \leqslant 0,
$$

при использовании которой из (15) следует неравенство

$$
I=-\frac{b}{a}\|\psi(x)\|_{0}^{2}-\frac{1}{a}\left\|\int_{0}^{x} \psi(\xi) d \xi\right\|_{0}^{2} \leqslant 0 .
$$

Для того чтобы получить соотношение между $\varphi(x)$ и $\psi(x)$, принесенное из области $\Omega^{-}$на линию $y=0$, выпишем решение задачи Коши $(7),(8)$ для уравнения (3) [2]:

$$
\begin{aligned}
& u(x, y)=\frac{1}{B(\alpha, \beta)} \int_{0}^{1} \varphi\left[x+\frac{2}{m+2}(-y)^{(m+2) / 2}(2 t-1)\right] t^{\beta-1}(1-t)^{\alpha-1} d t+ \\
& +\frac{y}{B(1-\alpha, 1-\beta)} \int_{0}^{1} \psi\left[x+\frac{2}{m+2}(-y)^{(m+2) / 2}(2 t-1)\right] \times \\
& \times t^{-\alpha}(1-t)^{-\beta} d t, \quad|c|<\frac{m}{2} \\
& u(x, y)=\varphi\left[x+\frac{2}{m+2}(-y)^{(m+2) / 2}\right]+ \\
& +\frac{2 y}{m+2} \int_{0}^{1} \psi\left[x+\frac{2}{m+2}(-y)^{(m+2) / 2}(2 t-1)\right](1-t)^{-\beta} d t, \quad c=\frac{m}{2} \\
& u(x, y)=\varphi\left[x-\frac{2}{m+2}(-y)^{(m+2) / 2}\right]+ \\
& +\frac{2 y}{m+2} \int_{0}^{1} \psi\left[x+\frac{2}{m+2}(-y)^{(m+2) / 2}(1-2 t)\right](1-t)^{-\alpha} d t, \quad c=-\frac{m}{2},
\end{aligned}
$$

где

$$
\alpha=\frac{m-2 c}{2(m+2)}, \quad \beta=\frac{m+2 c}{2(m+2)},
$$

а $B(\gamma, z)$ - бета-функция.

Учитывая условие (6), перепишем (19)-(21) в виде

$$
\begin{array}{ll}
h_{r}\left(\frac{r+x}{2}\right)=\frac{(r-x)^{1-\alpha-\beta} \Gamma(\beta)}{B(\alpha, \beta)} D_{r x}^{-\beta}\left[(r-\xi)^{\alpha-1} \varphi(\xi)\right]- & \\
-\frac{\Gamma(1-\alpha)}{B(1-\alpha, 1-\beta)}\left(\frac{m+2}{4}\right)^{2 /(m+2)} D_{r x}^{\alpha-1}\left[(r-\xi)^{-\beta} \psi(\xi)\right], & |c|<\frac{m}{2} ; \\
h_{r}\left(\frac{r+x}{2}\right)=\varphi(r)-\frac{1}{2}\left(\frac{m+2}{4}\right)^{-\beta} D_{r x}^{-1}\left[(r-\xi)^{-\beta} \psi(\xi)\right], \quad c=\frac{m}{2}
\end{array}
$$




$$
h_{r}\left(\frac{r+x}{2}\right)=\varphi(x)-\frac{\Gamma(1-\alpha)}{2}\left(\frac{m+2}{4}\right)^{-\alpha} D_{r x}^{\alpha-1} \psi(\xi), \quad c=-\frac{m}{2} .
$$

Для любой функции $v(x) \in L_{1}[0, r]$ справедливы следующие свойства дробного интегро-дифференцирования с одинаковыми началами [13]:

$$
\begin{gathered}
D_{r x}^{\alpha_{1}} D_{r \xi}^{-\beta_{1}} v(s)=D_{r x}^{\alpha_{1}-\beta_{1}} v(\xi), \quad 0<\alpha_{1} \leqslant \beta_{1} ; \\
D_{r x}^{\alpha_{1}}|\xi-r|^{\alpha_{1}+\beta_{1}} D_{r \xi}^{\beta_{1}} v(s)=|x-r|^{\beta_{1}} D_{r x}^{\alpha_{1}+\beta_{1}}|\xi-r|^{\alpha_{1}} v(\xi), \quad \beta_{1}<0,0 \leqslant \alpha_{1}<1,
\end{gathered}
$$

с применением которых из (22)-(24) функция $\psi(x)$ выражается следующим образом:

$$
\begin{gathered}
\psi(x)=\frac{d_{2}}{d_{1}} D_{r x}^{1-\alpha-\beta} \varphi(\xi)-\frac{(r-x)^{\beta}}{d_{1}} D_{r x}^{1-\alpha} h_{r}\left(\frac{r+\xi}{2}\right), \quad|c|<\frac{m}{2} \\
\psi(x)=-\frac{(r-x)^{\beta}}{2 d_{3}} h_{r}^{\prime}\left(\frac{r+x}{2}\right), \quad c=\frac{m}{2} \\
\psi(x)=\frac{1}{d_{4}} D_{r x}^{1-\alpha} \varphi(\xi)-\frac{1}{d_{4}} D_{r x}^{1-\alpha} h_{r}\left(\frac{r+\xi}{2}\right), \quad c=-\frac{m}{2}
\end{gathered}
$$

где

$$
\begin{gathered}
d_{1}=\left(\frac{m+2}{4}\right)^{2 /(m+2)} \frac{\Gamma(2-\alpha-\beta)}{\Gamma(1-\beta)}, \quad d_{2}=\frac{\Gamma(\alpha+\beta)}{\Gamma(\alpha)}, \\
d_{3}=\frac{1}{2}\left(\frac{m+2}{4}\right)^{-\beta}, \quad d_{4}=\frac{1}{2}\left(\frac{m+2}{4}\right)^{-\alpha} \Gamma(1-\alpha) .
\end{gathered}
$$

Подставляя найденные значения (25)-(27) функции $\psi(x)$ при $h_{r}(x)=0$, из (14) имеем:

$$
\begin{gathered}
I=\frac{d_{2}}{d_{1}} \int_{0}^{r} \varphi(x) D_{r x}^{1-\alpha-\beta} \varphi(\xi) d x, \quad|c|<\frac{m}{2} ; \\
I=0, \quad c=\frac{m}{2} ; \\
I=\frac{1}{d_{4}} \int_{0}^{r} \varphi(x) D_{r x}^{1-\alpha} \varphi(\xi) d x, \quad c=-\frac{m}{2} .
\end{gathered}
$$

Известно [24], что для любой абсолютно непрерывной на $[0, r]$ функции $v(x)$ при $v(r)=0$ справедливо неравенство

$$
v(x) D_{r x}^{\gamma} v(\xi) \geqslant \frac{1}{2} D_{r x}^{\gamma} v^{2}(\xi), \quad 0<\gamma \leqslant 1 .
$$

Тогда с учетом (31) и представления

$$
D_{r x}^{\gamma} v^{2}(\xi)=-\frac{1}{\Gamma(1-\gamma)} \int_{x}^{r} \frac{2 v(\xi) v^{\prime}(\xi)}{(\xi-x)^{\gamma}} d \xi
$$

из (28) и (30) имеем 


$$
\begin{gathered}
I \geqslant \frac{d_{2}}{2 d_{1}} \int_{0}^{r} D_{r x}^{1-\alpha-\beta} \varphi^{2}(\xi) d x= \\
=\frac{d_{2}}{2 d_{1} \Gamma(\alpha+\beta)} \int_{0}^{r} x^{\alpha+\beta-1} \varphi^{2}(x) d x \geqslant 0, \quad|c|<\frac{m}{2} \\
I \geqslant \frac{1}{2 d_{4}} \int_{0}^{r} D_{r x}^{1-\alpha} \varphi^{2}(\xi) d x=\frac{1}{2 d_{4} \Gamma(\alpha)} \int_{0}^{r} x^{\alpha-1} \varphi^{2}(x) d x \geqslant 0, \quad c=-\frac{m}{2}
\end{gathered}
$$

Таким образом, из (32) и (33) получаем, что $I \geqslant 0$. С другой стороны, из (18) имеем, что $I \leqslant 0$. Следовательно, $I=0$ при $-m / 2 \leqslant c<m / 2$, которое имеет место тогда и только тогда, когда $\varphi(x)=0$. Тогда, как видно из $(25)$ и $(27)$, функция $\psi(x)=0$. При $c=m / 2$ из (18) и $(29)$ следует, что $\psi(x)=0$, и тогда из $(13)$ получаем, что и $\varphi(x)=0$. При этом из формул $(19)-(21)$ имеем $u(x, y) \equiv 0$ в области $\Omega^{-}$.

В области $\Omega^{+}$задача для уравнения (1) совпадает со второй краевой задачей для уравнения Аллера, решение которого выписывается в виде [25]:

$$
\begin{aligned}
u(x, y)=\int_{0}^{r}[\varphi(\xi) & \left.-b \varphi^{\prime \prime}(\xi)\right] G(x, y ; \xi, 0) d \xi+ \\
+\int_{0}^{y} G(x, y ; r, \eta) & {\left[a \nu_{r}(\eta)+b \nu_{r}^{\prime}(\eta)\right] d \eta-} \\
& -\int_{0}^{y} G(x, y ; 0, \eta)\left[a \nu(\eta)+b \nu^{\prime}(\eta)\right] d \eta,
\end{aligned}
$$

где

$$
\begin{aligned}
G(x, y ; \xi, \eta)=\frac{1}{r}+\frac{2}{r} \sum_{n=1}^{\infty} \frac{1}{1+b \mu_{n}} & \exp \left(-\frac{a \mu_{n}}{1+b \mu_{n}}(y-\eta)\right) \times \\
& \times \cos \left(\sqrt{\mu_{n}} \xi\right) \cos \left(\sqrt{\mu_{n}} x\right), \mu_{n}=\left(\frac{\pi n}{r}\right)^{2} .
\end{aligned}
$$

При однородных условиях (4), (5) и (7) из представления (34) находим, что $u(x, y) \equiv 0$ в области $\Omega^{+}$. Таким образом, однородная задача соответствующая задаче (4)-(6) для уравнения (1), имеет только тривиальное решение, откуда следует единственность регулярного решения исследуемой задачи.

Существование решения. Перейдем к доказательству существования решения исследуемой задачи. Из (22)-(24) верно, что

$$
\begin{aligned}
\varphi(x)=\frac{d_{1}}{d_{2}} D_{r x}^{\alpha+\beta-1} \psi(\xi)+ & \\
& +\frac{(r-x)^{1-\alpha}}{d_{2}} D_{r x}^{\beta}\left[(r-\xi)^{\alpha+\beta-1} h_{r}\left(\frac{r+\xi}{2}\right)\right], \quad|c|<\frac{m}{2} \\
\varphi(r)= & d_{3} D_{r x}^{-1}\left[(r-\xi)^{-\beta} \psi(\xi)\right]+h_{r}\left(\frac{r+x}{2}\right), \quad c=\frac{m}{2}
\end{aligned}
$$




$$
\varphi(x)=d_{4} D_{r x}^{\alpha-1} \psi(\xi)+h_{r}\left(\frac{r+x}{2}\right), \quad c=-\frac{m}{2} .
$$

Исключая функцию $\varphi(x)$ из (13) и соотношений $(35)-(37)$, относительно функции $\psi(x)$ получим следующие равенства:

$$
\begin{gathered}
\psi(x)+\frac{a d_{1}}{b d_{2}} D_{r x}^{\alpha+\beta-1} \psi(\xi)-\frac{1}{b} D_{r x}^{-2} \psi(\xi)=f_{\beta}(x), \quad|c|<\frac{m}{2} \\
\psi(x)=-\frac{(r-x)^{\beta}}{2 d_{3}} h_{r}^{\prime}\left(\frac{r+x}{2}\right), \quad c=\frac{m}{2} \\
\psi(x)+\frac{a}{b d_{4}} D_{r x}^{\alpha-1} \psi(\xi)-\frac{1}{b} D_{r x}^{-2} \psi(\xi)=f_{0}(x), \quad c=-\frac{m}{2}
\end{gathered}
$$

где

$$
\begin{aligned}
f_{\beta}(x)=\frac{a}{b} h_{r}(r)-(r-x)\left[\frac{a}{b} \nu_{r}(0)\right. & \left.+\nu_{r}^{\prime}(0)\right]- \\
& -\frac{a(r-x)^{1-\alpha}}{b d_{2}} D_{r x}^{\beta}\left[(r-\xi)^{\alpha+\beta-1} h_{r}\left(\frac{r+\xi}{2}\right)\right] .
\end{aligned}
$$

С учетом равенства

$$
D_{r r-x}^{\gamma} \psi(\xi)=D_{0 x}^{\gamma} \psi(r-\xi), \quad \gamma<0
$$

после замены $x$ на $(r-x)$, когда $\psi(r-x)=q(x)$ и $f_{\beta}(r-x)=w(x)$, из (38) получим

$$
q(x)+\frac{a d_{1}}{b d_{2}} D_{0 x}^{\alpha+\beta-1} q(\xi)-\frac{1}{b} D_{0 x}^{-2} q(\xi)=w(x) .
$$

Уравнение (41) представляет собой уравнение Вольтерра второго рода типа свертки. Следовательно, при $w(x) \in L_{1}[0, r]$ оно имеет единственное решение.

В силу представления оператора дробного интегрирования перепишем уравнение (41) в виде

$$
q(x)+\frac{a d_{1}}{b d_{2}}\left(q(x) * x^{-\alpha-\beta}\right)-\frac{1}{b}(q(x) * x)=(x * W(x)) .
$$

Здесь $(f(x) * g(x))=\int_{0}^{x} f(x-\xi) g(\xi) d \xi$ - свертка функций $f(x)$ и $g(x) ; W(x)=$ $=D_{0 x}^{2} w(\xi)$.

Пусть $Q(p)$ и $W^{*}(p)$ являются изображениями функций $q(x)$ и $W(x)$ по Лапласу:

$$
q(x) \risingdotseq Q(p), \quad W(x) \risingdotseq W^{*}(p) .
$$

Применяя преобразование Лапласа, из (42) находим

$$
Q(p)=\frac{W^{*}(p)}{\Delta(p) p^{2}}
$$

где

$$
\Delta(p)=1+\frac{a d_{1}}{b d_{2} p^{1-\alpha-\beta}}-\frac{1}{b p^{2}}
$$


Так как при достаточно больших значениях $p$ имеет место равенство

$$
\int_{0}^{\infty} e^{-\Delta(p) t} d t=\frac{1}{\Delta(p)}
$$

равенство (43) можно переписать в виде

$$
Q(p)=\int_{0}^{\infty} \frac{e^{-\Delta(p) t}}{p^{2}} W^{*}(p) d t
$$

Найдем обратное преобразование Лапласа к уравнению (44). Для этого воспользуемся известной формулой $x^{k-1} \phi\left(\gamma, k ; z x^{\gamma}\right) \risingdotseq p^{-k} e^{z p^{-\gamma}}[26]$ :

$$
q(x)=\int_{0}^{\infty} e^{-t}\left[W(x) * \phi\left(1-\alpha-\beta ; 1 ;-\frac{a d_{1}}{b d_{2}} t x^{1-\alpha-\beta}\right) * \phi\left(2 ; 1 ; \frac{1}{b} t x^{2}\right)\right] d t
$$

где

$$
\phi(\gamma, k ; t)=\sum_{n=0}^{\infty} \frac{t^{n}}{n ! \Gamma(n \gamma+k)}
$$

- функция Райта.

Воспользовавшись обозначениями, введенными в работе [27]:

$$
\begin{gathered}
G_{n}^{k} \equiv G_{n}^{k}\left(x ; \lambda_{1}, \ldots, \lambda_{n} ; \gamma_{1}, \ldots, \gamma_{n}\right)=\int_{0}^{\infty} e^{-t} S_{n}^{k}\left(x ; \lambda_{1} t, \ldots, \lambda_{n} t ; \gamma_{1}, \ldots, \gamma_{n}\right) d t \\
S_{n}^{k}\left(x ; \lambda_{1} t, \ldots, \lambda_{n} t ; \gamma_{1}, \ldots, \gamma_{n}\right)=\left(h_{1} * h_{2} * \cdots * h_{n}\right)(x), \\
h_{i}=h_{i}(x)=x^{k_{i}-1} \phi\left(\gamma_{i}, k_{i} ; \lambda_{i} t x^{\gamma_{i}}\right), \quad i=1,2, \ldots, n ; \quad k=\sum_{i=1}^{n} k_{i},
\end{gathered}
$$

решение (45) можно переписать в виде

$$
\begin{aligned}
\psi(r-x)=q(x)=\int_{0}^{\infty} e^{-t}\left[W(x) * S_{2}^{2}\left(x ;-\frac{a d_{1}}{b d_{2}} t, \frac{1}{b} t ; 1-\alpha-\beta, 2\right)\right] d t= \\
=\int_{0}^{x} W(\xi) G_{2}^{2}\left(x-\xi ;-\frac{a d_{1}}{b d_{2}}, \frac{1}{b} ; 1-\alpha-\beta, 2\right) d \xi
\end{aligned}
$$

Таким образом, переобозначив в последнем равенстве $(r-x)$ через $x$ и выполнив подстановку $t=r-\xi$ под знаком интеграла, получим

$$
\begin{aligned}
& \psi(x)=\int_{0}^{r-x} w^{\prime \prime}(\xi) G_{2}^{2}(r\left.-x-\xi ;-\frac{a d_{1}}{b d_{2}}, \frac{1}{b} ; 1-\alpha-\beta, 2\right) d \xi= \\
&=\int_{x}^{r} f_{\beta}^{\prime \prime}(t) G_{2}^{2}\left(t-x ;-\frac{a d_{1}}{b d_{2}}, \frac{1}{b} ; 1-\alpha-\beta, 2\right) d t .
\end{aligned}
$$

При $\beta=0$ из (46) получаем решение уравнения (40) в виде

$$
\psi(x)=\int_{r}^{x} f_{0}^{\prime \prime}(t) G_{2}^{2}\left(t-x ;-\frac{a d_{1}}{b}, \frac{1}{b} ; 1-\alpha, 2\right) d t .
$$


Из (13), (35) и (37) при подстановке формул (39), (46) и (47) функция $\varphi(x)$ находится однозначно. Следовательно, после нахождения функций $\varphi(x)$ и $\psi(x)$ решение задачи (4)-(6) для уравнения $(2)$ в области $\Omega^{+}$находится по формуле (34), а в области $\Omega^{-}$решение задачи Коши для уравнения (3) выписывается по одной из формул (19)-(21).

Конкурирующие интересы. Конкурирующих интересов не имею.

Авторская ответственность. Я несу полную ответственность за предоставление окончательной версии рукописи в печать. Окончательная версия рукописи мною одобрена.

Финансирование. Исследование выполнялось без финансирования.

\section{Библиографический список}

1. Hallaire M. Potential efficace de l'eau dans le sol en régime de dessèchement / Assemblée générale de Berkeley=General Assembly of Berkeley, Publ. no. 62 (August 1963). Gentbrugge, 1963. pp. 114-122, http://hydrologie.org/redbooks/a062/iahs_062_0114.pdf.

2. Смирнов М. М. Вырождающиеся гиперболические уравнения. Минск: Вышэйшая школа, 1977. 158 с.

3. Showalter R.E., Ting T.W. Pseudoparabolic partial differential equations // SIAM J. Math. Anal., 1970. vol. 1, no. 1. pp. 1-26. doi : 10.1137/0501001.

4. Чудновский А. Ф. Теплофизика почв. М.: Наука, 1976. 352 с.

5. Нахушев А. М. Об одном классе нагруженных уравнений в частных производных дробного порядка // Доклады Адыгской (Черкесской) Международной академии наук, 2012. Т. 14, № 1. С. 51-57.

6. Баренблатт Г. И., Желтов И. П., Кочина И. Н. Об основных представлениях теории фильтрации однородных жидкостей в трещиноватых породах // ПMМ, 1960. Т. 24, № 5. C. $852-864$.

7. Coleman B. D., Duffin R. J., Mizel V. J. IInstability, uniqueness, and nonexistence theorems for the equation $u_{t}=u_{x x}-u_{x t x}$ on a strip // Arch. Rat. Mech. Anal., 1965. vol. 19, no. 2. pp. 100-116. doi: 10.1007/BF00282277.

8. Colton D. Pseudoparabolic equations in one space variable// J. Differ. Equations, 1972. vol. 12, no. 3. pp. 559-565. doi: 10.1016/0022-0396 (72)90025-3.

9. Янгарбер В. А. О смешанной задаче для модифицированного уравнения влагопереноса // ПМТФ, 1967. №1. С. 91-96.

10. Шхануков М. Х. О некоторых краевых задачах для уравнения третьего порядка, возникающих при моделировании фильтрации жидкости в пористых средах // Дифферени. уравнения, 1982. Т. 18, № 4. С. 689-699.

11. Водахова В. А. Краевая задача с нелокальным условием А. М. Нахушева для одного псевдопараболического уравнения влагопереноса // Дифферени. уравнения, 1982. Т. 18, № 2. С. 280-285.

12. Кожанов А. И. Об одной нелокальной краевой задаче с переменными коэффициентами для уравнений теплопроводности и Аллера // Дифферени. уравнения, 2004. Т. 40, № 6 . C. $763-774$.

13. Нахушев А. М. Уравнения математической биологии. М.: Высшая школа, 1995. 301 с.

14. О. А. Репин Аналог задачи Нахушева для уравнения Бицадзе-Лыкова // Дифферени. уравнения, 2002. Т. 38, № 10. С. 1412-1417.

15. Репин О. А., Кумыкова С. К. Нелокальная задача для уравнения Бицадзе-Лыкова // Изв. вузов. Матем., 2010. № 3. С. 28-35.

16. Нахушев А. М. Дробное исчисление и его применение. М.: Физматлит, 2003. 272 с. 
17. Кальменов Т. Ш. Критерий единственности решения задачи Дарбу для одного вырождающегося гиперболического уравнения // Дифберени. уравнения, 1971. Т. 7, № 1. C. $178-181$.

18. Кальменов Т. Ш. О задаче Дарбу для одного вырождающегося уравнения // Дифференц. уравнения, 1974. Т. 10, №1. С. 59-68.

19. Л. С. Пулькина Об одной нелокальной задаче для вырождающегося гиперболического уравнения // Матем. заметки, 1992. Т. 51, № 3. С. 91-96.

20. О. А. Репин, С. К. Кумыкова Задача со смещением для вырождающегося внутри области гиперболического уравнения // Вестн. Сам. гос. техн. ун-та. Сер. Физ.-мат. науки, 2014. № 1(34). С. 37-47. doi : 10.14498/vsgtu1280.

21. Балкизов Ж. А. Первая краевая задача для вырождающегося внутри области гиперболического уравнения // Владикавк. матем. журн., 2016. Т. 18, № 2. С. 19-30.

22. Балкизов Ж. А. Краевая задача для вырождающегося внутри области гиперболического уравнения // Известия вузов. Северо-Кавказский регион. Серия: Естественные науки, 2016. № 1. С. 5-10. doi: 10.18522/0321-3005-2016-1-5-10.

23. Фихтенгольц Г. М. Курс дифференииального и интегрального исчисления. Т. 1. М.: Физматлит, 2003. 680 с.

24. Ж. А. Балкизов Первая краевая задача для уравнения параболо-гиперболического типа третьего порядка с вырождением типа и порядка в области гиперболичности // Уфимск. матем. журн., 2017. Т. 9, № 2. С. 25-39.

25. Макаова Р.Х. Вторая краевая задача для обобщенного уравнения Аллера с дробной производной Римана-Лиувилля // Докладъ Адыгской (Черкесской) Международной академии наук, 2015. Т. 17, №3. С. 35-38.

26. Wright E. M. The generalized Bessel function of order greater than one// Quart. J. Math. Oxford Ser., 1940. vol. os-11, no. 1. pp. 36-48. doi : 10.1093/qmath/os-11.1.36.

27. Псху А. В. Начальная задача для линейного обыкновенного дифференциального уравнения дробного порядка // Матем. сб., 2011. Т. 202, №4. С. 111-122. doi: 10.4213/ $\operatorname{sm} 7645$. 
MSC: 35L25, 35L80

\title{
A boundary value problem for a third order hyperbolic equation with degeneration of order inside the domain
}

\author{
R. Kh. Makaova \\ Institute of Applied Mathematics and Automation \\ of Kabardin-Balkar Scientific Centre of RAS, \\ 89 a, Shortanova st., Nal'chik, 360000, Russian Federation.
}

\begin{abstract}
In this paper we study the boundary value problem for a degenerating third order equation of hyperbolic type in a mixed domain. The equation under consideration in the positive part of the domain coincides with the Hallaire equation, which is a pseudoparabolic type equation. Moreover, in the negative part of the domain it coincides with a degenerating hyperbolic equation of the first kind, the particular case of the Bitsadze-Lykov equation. The existence and uniqueness theorem for the solution is proved. The uniqueness of the solution to the problem is proved with the Tricomi method. Using the functional relationships of the positive and negative parts of the domain on the degeneration line, we arrive at the convolution type Volterra integral equation of the 2 nd kind with respect to the desired solution by a derivative trace. With the Laplace transform method, we obtain the solution of the integral equation in its explicit form. At last, the solution to the problem under study is written out explicitly as the solution of the second boundary-value problem in the positive part of the domain for the Hallaire equation and as the solution to the Cauchy problem in the negative part of the domain for a degenerate hyperbolic equation of the first kind.
\end{abstract}

Keywords: boundary-value problem, third order hyperbolic equation, Hallaire equation.

Received: $27^{\text {th }}$ October, $2017 /$ Revised: $11^{\text {th }}$ December, $2017 /$ Accepted: $18^{\text {th }}$ December, $2017 /$ First online: $28^{\text {th }}$ December, 2017

Competing interests. I have no competing interests.

Author's Responsibilities. I take full responsibility for submitting the final manuscript in print. I approved the final version of the manuscript.

\section{Research Article}

(우(i) The content is published under the terms of the Creative Commons Attribution 4.0 International License (http://creativecommons.org/licenses/by/4.0/)

Please cite this article in press as:

Makaova R. Kh. A boundary value problem for a third order hyperbolic equation with degeneration of order inside the domain, Vestn. Samar. Gos. Tekhn. Univ., Ser. Fiz.-Mat. Nauki [J. Samara State Tech. Univ., Ser. Phys. Math. Sci.], 2017, vol. 21, no. 4, pp. 651-664. doi: 10.14498/vsgtu1574 (In Russian).

\section{Author's Details:}

Ruzanna Kh. Makaova (10) http://orcid.org/0000-0003-4095-2332

Junior Researcher; Dept. of Mixed Type Equations; e-mail: makaova.ruzanna@mail.ru 
Funding. The research has not had any funding.

\section{References}

1. Hallaire M. Potential efficace de l'eau dans le sol en régime de dessèchement, In: Assemblée générale de Berkeley=General Assembly of Berkeley, Publ. no. 62 (August 1963). Gentbrugge, 1963, pp. 114-122, http://hydrologie.org/redbooks/a062/iahs_062_0114.pdf.

2. Smirnov M. M. Vyrozhdaiushchiesia giperbolicheskie uravneniia [Degenerate hyperbolic equations]. Minsk, Vysheishaia shkola, 1977, 158 pp. (In Russian)

3. Showalter R.E., Ting T.W. Pseudoparabolic partial differential equations, SIAM J. Math. Anal., 1970, vol. 1, no. 1, pp. 1-26. doi: 10.1137/0501001.

4. Chudnovskii A. F. Teplofizika pochv [Thermal Physics of Soils]. Moscow, Nauka, 1976, 352 pp. (In Russian)

5. Nakhushev A. M. On a class of loaded partial differential equations of fractional order, Doklady Adygskoi (Cherkesskoi) Mezhdunarodnoi akademii nauk, 2012, vol. 14, no. 1, pp. 5157 (In Russian).

6. Barenblatt G. I., Zheltov Iu. P., Kochina I. N. Basic concepts in the theory of seepage of homogeneous liquids in fissured rocks [strata], J. Appl. Math. Mech., 1960, vol. 24, no. 5, pp. 1286-1303. doi : 10.1016/0021-8928(60)90107-6.

7. Coleman B. D., Duffin R. J., Mizel V. J. IInstability, uniqueness, and nonexistence theorems for the equation $u_{t}=u_{x x}-u_{x t x}$ on a strip, Arch. Rat. Mech. Anal., 1965, vol. 19, no. 2, pp. 100-116. doi: 10.1007/BF00282277:en.

8. Colton D. Pseudoparabolic equations in one space variable, J. Differ. Equations, 1972, vol. 12, no. 3, pp. 559-565. doi: 10.1016/0022-0396(72)90025-3.

9. Yangarber V. A. The mixed problem for a modified moisture-transfer equation, J. Appl. Mech. Tech. Phys., 1967, vol.8, no. 1, pp. 62-64. doi: 10.1007/BF00913245.

10. Shkhanukov M. Kh. Some boundary value problems for a third-order equation that arise in the modeling of the filtration of a fluid in porous media, Differ. Uravn., 1982, vol. 18, no. 4, pp. 689-699 (In Russian).

11. Vogahova V. A. A boundary value problem with A. M. Nakhushev's nonlocal condition for a pseudoparabolic equation of moisture transfer, Differ. Uravn., 1982, vol. 18, no. 2, pp. 280-285 (In Russian).

12. Kozhanov A. I. On a Nonlocal Boundary Value Problem with Variable Coefficients for the Heat Equation and the Aller Equation, Differ. Equ., 2004, vol.40, no. 6, pp. 815-826. doi : 10.1023/B:DIEQ.0000046860.84156.f0.

13. Nakhushev A. M. Uravneniia matematicheskoi biologii [Equations of Mathematical Biology]. Moscow, Vysshaia shkola, 1995, 301 pp. (In Russian)

14. Repin O. A. An Analog of the Nakhushev Problem for the Bitsadze-Lykov Equation, Differ. Equ., 2002, vol.38, no. 10, pp. 1503-1509. doi: 10.1023/A:1022339217281.

15. Repin O. A., Kumykova S. K. A nonlocal problem for the Bitsadze-Lykov equation, Russian Math. (Iz. VUZ), 2010, vol. 54, no. 3, pp. 24-30. doi: 10.3103/S1066369X10030059.

16. Nakhushev A. M. Drobnoe ischislenie $i$ ego primenenie [Fractional calculus and its application]. Moscow, Fizmatlit, 2003, 272 pp. (In Russian)

17. Kal'menov T. Sh. A criterion for the uniqueness of the solution of the Darboux problem for a certain degenerate hyperbolic equation, Differ. Uravn., 1971, vol. 7, no. 1, pp. 178-181 (In Russian).

18. Kal'menov T. Sh. The Darboux problem for a certain degenerate equation, Differ. Uravn., 1974, vol. 10, no. 1, pp. 59-68 (In Russian).

19. Pul'kina L. S. Certain nonlocal problem for a degenerate hyperbolic equation, Math. Notes, 1992, vol. 51, no. 3, pp. 286-290. doi: 10.1007/BF01206393.

20. Repin O. A., Kumykova S. K. A Boundary-value Problem with Shift for a Hyperbolic Equation Degenerate in the Interior of a Region, Vestn. Samar. Gos. Tekhn. Univ., Ser. Fiz.-Mat. Nauki [J. Samara State Tech. Univ., Ser. Phys. Math. Sci.], 2014, no. 1(34), pp. 3747 (In Russian). doi: 10.14498/vsgtu1280. 
21. Balkizov Zh. A. The first boundary value problem for a degenerate hyperbolic equation, Vladikavkaz. Mat. Zh., 2016, vol. 18, no. 2, pp. 19-30 (In Russian).

22. Balkizov $\mathrm{Zh}$. A. The boundary-value problem for a degenerate hyperbolic equation in the area, Izvestiya Vuzov. Severo-Kavkazskii Region. Natural Science, 2016, no. 1, pp. 5-10 (In Russian). doi: 10.18522/0321-3005-2016-1-5-10.

23. Fikhtengol'ts G. M. Kurs differentsial'nogo i integral'nogo ischisleniia [Course of Differential and Integral Calculus], vol. 1. Moscow, Fizmatlit, 2003, 680 pp. (In Russian)

24. Balkizov Zh. A. Dirichlet boundary value problem for a third order parabolic-hyperbolic equation with degenerating type and order in the hyperbolicity domain, Ufa Math. Journal, 2017, vol. 9, no. 2, pp. 25-39. doi : 10.13108/2017-9-2-25.

25. Makaova R.Kh. The second boundary-value problem for generalized Hallaire equation with fractional Riemann-Liouville derivative, Doklady Adygskoi (Cherkesskoi) Mezhdunarodnoi akademii nauk, 2015, vol. 17, no. 3, pp. 35-38 (In Russian).

26. Wright E. M. The generalized Bessel function of order greater than one, Quart. J. Math. Oxford Ser., 1940, vol. os-11, no. 1, pp. 36-48. doi : 10.1093/qmath/os-11.1.36.

27. Pskhu A. V. Initial-value problem for a linear ordinary differential equation of noninteger order, Sb. Math., 2011, vol. 202, no. 4, pp. 571-582. doi: 10.1070/SM2011v202n04ABEH004156. 